\title{
Vinyl Polymerization. CCLXXI. Polymerization of Acrylonitrile Initiated by the System of Tetramethyltetrazene and 1-Chloro-2, 4-dinitrobenzene
}

\author{
Kazuo Sugiyama, Tadao Nakaya, and Minoru Imoto \\ Department of Applied Chemistry, Faculty of Engineering, \\ Osaka City University, Sugimoto-cho, \\ Sumiyoshi-ku, Osaka, Japan.
}

(Received February 12, 1971)

\begin{abstract}
The polymerization of acrylonitrile(AN) initiated by a $\sigma$-complex of tetramethyltetrazene(TMT) and 1-chloro-2,4-dinitrobenzene(DNCB) was carried out in dimethylformamide(DMF). The molar ratio of TMT and DNCB in the complex was spectroscopically determined to be $1: 1$. The polymerization was confirmed to proceed through a radical mechanism. The rate of polymerization was found to be 0.6 -order in [TMT], approximately 0.5 -order in [DNCB], and second-order in [AN]. The overall activation energy for the polymerization was estimated as $17.8 \mathrm{kcal} / \mathrm{mol}$. A kinetic reaction scheme and a possible initiation mechanism for the polymerization are proposed to explain the experimental results.
\end{abstract}

KEY WORDS Acrylonitrile / Tetramethyltetrazene-1-chloro-2,4-dinitrobenzene System / Radical Polymerization / Kinetic Scheme / $\sigma^{-}$ Complex /

In 1959, McGowan ${ }^{1}$, et al., stated in their patent that tetramethyltetrazene(TMT) can initiate the polymerization of ethylene. Recently we made a kinetic study on the polymerization of acrylonitrile initiated with $\mathrm{TMT}^{2}$, and found that the rate of polymerization of vinyl monomer (acrylonitrile, methyl methacrylate or styrene) initiated by TMT was remarkably promoted by the presence of a small amount of acetic anhydride $^{2}$, benzyl chloride ${ }^{3}$ or dimethyl maleate ${ }^{4}$. The active species for the initiation was concluded to be dimethylamino radical which is formed during the reaction of TMT with the promotor mentioned above.

TMT is a strong base, while the carbon atom at the 1-position of 1-halogeno-2,4-dinitrobenzene is strongly electrophilic. Accordingly the reaction of TMT with 1-halogeno-2,4-dinitrobenzene may easily occur. Thus the polymerization of vinyl monomer by the system of TMT and 1halogeno-2,4-dinitrobenzene is expected to give an interesting result.

The present paper deals with the homogeneous polymerization of acrylonitrile by TMT in the presence of 1-halogeno-2,4-dinitrobenzene in di-
methylformamide(DMF). It became clear that the 1-halogeno-2,4-dinitrobenzene can promote remarkably the initiation by TMT. The order of the promoting activities of the 1-halogeno-2, 4-dinitrobenzene was as follows:

$$
\begin{aligned}
\text { F- } \mathrm{C}_{6} \mathrm{H}_{3}\left(\mathrm{NO}_{2}\right)_{2}>\mathrm{Cl}-\mathrm{C}_{6} \mathrm{H}_{3}\left(\mathrm{NO}_{2}\right)_{2} \\
\quad>\mathrm{Br}-\mathrm{C}_{6} \mathrm{H}_{3}\left(\mathrm{NO}_{2}\right)_{2}>\mathrm{I}-\mathrm{C}_{6} \mathrm{H}_{3}\left(\mathrm{NO}_{2}\right)_{2}
\end{aligned}
$$

In order to make a kinetic study, 1-chloro-2, 4-dinitrobenzene(DNCB) was selected. In Discussion (below), a mechanism for the initiating reaction is proposed. Further, the reaction of TMT with DNCB was also examined, including the formation of a $\sigma$-complex of TMT and DNCB.

\section{EXPERIMENTAL}

\section{Materials}

1-Fluoro-2,4-dinitrobenzene, 1-chloro-2,4-dinitrobenzene(DNCB), and 1,1-dimethylhydrazine were of commercial origin and were purified by distillation or crystallization. By the substitution reaction of DNCB with sodium iodide, 1iodo-2,4-dinitrobenzene was prepared ${ }^{5}$. 1-Bromo2,4-dinitrobenzene was likewise synthesized from DNCB and sodium bromide. TMT was prepared 
by the oxidation of the 1,1-dimethylhydrazine with mercuric oxide, according to Bull, et al. ${ }^{6}$ Acrylonitrile(AN), methyl methacrylate(MMA), styrene $(\mathrm{St})$, and solvents were purified in the usual manner and redistilled under a stream of nitrogen before use.

\section{Polymerization Procedure}

The required amounts of monomer and initiator were placed in a tube, cooled in a dry icemethanol bath and sealed in vacuo. The sealed tube was shaken in a thermostat maintained at a definite temperature. After polymerization for a given time, the content of the tube was poured into a large amount of methanol to precipitate the polymer. The resulting polymer was purified twice by the usual reprecipitation method. The rate of conversion was calculated from the weight of the dry polymer obtained.

\section{Characterization of the Polymer}

Chemical composition of the resulting polymers was determined from their elemental analyses. The intrinsic viscosities $(\mathrm{d} l / \mathrm{g})$ of the polyacrylonitrile were determined in DMF at $30^{\circ} \mathrm{C}$, using an Ubbelohde viscometer. According to Houtz ${ }^{7}$, the number-average molecular weights of polymers, $M_{n}$, were calculated from the intrinsic viscosity $[\eta]$ values.

$$
[\eta](\mathrm{d} l / \mathrm{g})=1.75 \times 10^{-0.66} M_{n}
$$

UV Measurement

UV experiment was performed on a HitachiEPS-3T-type spectrophotometer.

\section{ESR Measurement}

ESR experiment was carried out with a JES$\mathrm{ME}$ instrument of Jeol Co. equipped with $100-\mathrm{H}_{z}$ modulation.

\section{RESULTS}

Polymerization of AN Initiated by TMT and Various 2,4-Dinitrohalogenobenzene Systems

The results of the polymerizations of $\mathrm{AN}$ by TMT and various 1-halogeno-2,4-dinitrobenzene are shown in Figure 1. The 1-halogeno-2,4dinitrobenzene used were 1-fluoro-2,4-dinitrobenzene, 1-chloro-2,4-dinitrobenzene, 1-bromo-2, 4-dinitrobenzene, and 1-iodo-2,4-dinitrobenzene.

It can be seen from Figure 1 that the catalytic activities for the polymerization of AN were

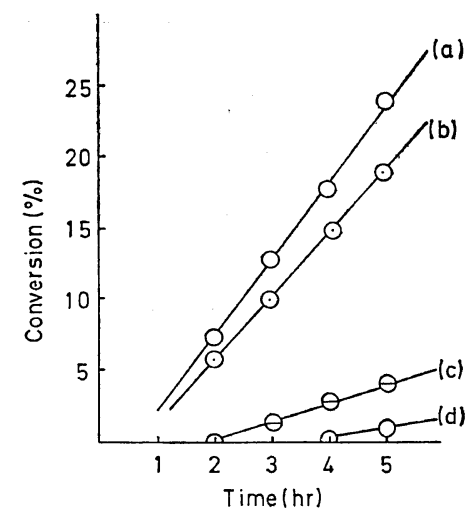

Figure 1. Conversion vs. time, in the polymerization of AN initiated by TMT and 1-halogeno-2, 4-dinitrobenzene at $80^{\circ} \mathrm{C}$. [AN], $4.56 \mathrm{~mol} / l$, [TMT], $2.0 \times 10^{-2} \mathrm{~mol} / l$, [1-halogeno-2,4-dinitrobenzene], $0.5 \times 10^{-2} \mathrm{~mol} / \mathrm{l}$ : (a) 1-ffuoro-2,4-dinitrobenzene; (b) 1-chloro-2,4-dinitrobenzene; (c) 1-bromo-2,4-dinitrobenzene; (d) 1-iodo-2,4-dinitrobenzene.

increased as follows.

$$
\begin{aligned}
& \mathrm{F}-\mathrm{C}_{6} \mathrm{H}_{3}\left(\mathrm{NO}_{2}\right)_{2}>\mathrm{Cl}-\mathrm{C}_{6} \mathrm{H}_{3}\left(\mathrm{NO}_{2}\right)_{2} \\
& \quad>\mathrm{Br}-\mathrm{C}_{6} \mathrm{H}_{3}\left(\mathrm{NO}_{2}\right)_{2}>\mathrm{I}-\mathrm{C}_{6} \mathrm{H}_{3}\left(\mathrm{NO}_{2}\right)_{2}
\end{aligned}
$$

The resulting polymers were confirmed to be homopolymers of AN by elementary analyses and the IR spectra.

\section{Confirmation of Radical Mechanism}

A bulk copolymerization of MMA with $\mathrm{St}$ was carried out at $70^{\circ} \mathrm{C}$.

Figure 2 shows the composition curve of the copolymer obtained, which is characteristic of a radical copolymerization. Furthermore, hydro-

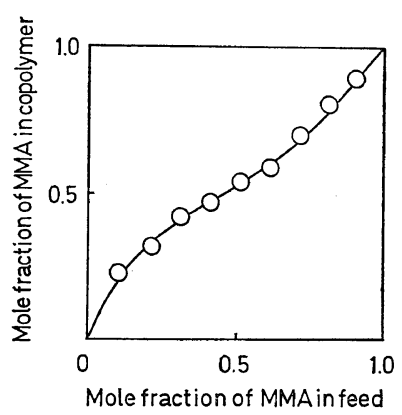

Figure 2. Composition curve of the copolymer of MMA with styrene which was obtained in bulk at $70^{\circ} \mathrm{C}$ for $5 \mathrm{hr}$ : [TMT], $2.0 \times 10^{-2} \mathrm{~mol} / l$; [DNCB], $0.5 \times 10^{-2} \mathrm{~mol} / \mathrm{l}$. 
quinone was found to act as an inhibitor for the polymerization of AN initiated by the TMT -DNCB system as shown in Figure 3.

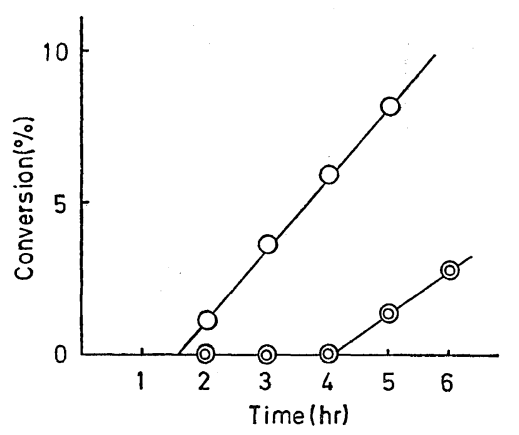

Figure 3. Effect of hydroquinone on the polymerization of AN initiated by TMT and DNCB: [AN], $4.56 \mathrm{~mol} / l$, [TMT], $2.0 \times 10^{-2} \mathrm{~mol} / l$, [DNCB], 0.5 $\times 10^{-2} \mathrm{~mol} / l$, at $80^{\circ} \mathrm{C}$ in DMF; $\odot$, [hydroquinone], $0.25 \times 10^{-3} \mathrm{~mol} / l, \bigcirc$, without hydroquinone.

From the copolymerization of MMA with St and the inhibition effect of hydroquinone, the polymerization was concluded to proceed via a free radical mechanism.

\section{Kinetics of Polymerization}

The polymerization of AN initiated by the TMT-DNCB system was studied kinetically. At first, the homogenous polymerization was carried out in DMF, varying the concentration of AN from 1.52 to $4.56 \mathrm{~mol} / \mathrm{l}$, and keeping the concentration of TMT and DNCB constant. From Figure 4, the existence of an induction

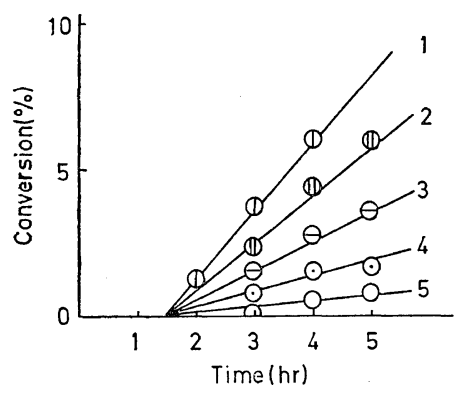

Figure 4. Conversion $v s$. time, in the polymerization of AN with the TMT-DNCB system in $\mathrm{DMF}$ at $80^{\circ} \mathrm{C}$ : [TMT], $2.0 \times 10^{-2} \mathrm{~mol} / l$, [DNCB], $0.5 \times 10^{-2} \mathrm{~mol}[l ;$; (1) $[\mathrm{AN}], 4.56$; (2) $[\mathrm{AN}], 3.80$; (3) [AN], 3.04; (4) [AN], 2.28; (5) [AN], $1.52 \mathrm{~mol} / l$. period was clearly observed.

The polymerization was also carried out in DMF, varying the concentration of TMT, while the concentrations of the monomer and DNCB were kept constant.

Furthermore, the polymerization was carried out in DMF by varying the concentration of DNCB. The results gained hitherto are tabulated in Table I.

Table I. The rate of polymerization $\left(R_{\mathrm{p}}\right)$ and number-average degree of polymerization $\left(P_{\mathrm{n}}\right)$ of the polymer results in the polymerization of $\mathrm{AN}$ in $\mathrm{DMF}$ at $80^{\circ} \mathrm{C}$ with the TMT-DNCB system

\begin{tabular}{ccccc}
\hline $\begin{array}{c}{[\mathrm{AN}],} \\
\mathrm{mol} / l\end{array}$ & $\begin{array}{c}{[\mathrm{TMT}] \times 10^{2},} \\
\mathrm{~mol} / l\end{array}$ & $\begin{array}{c}{[\mathrm{DNCB}] \times 10^{2},} \\
\mathrm{~mol} / l\end{array}$ & $\begin{array}{c}R_{\mathrm{p}} \times 10^{5}, \\
\mathrm{~mol} / l \mathrm{sec}\end{array}$ & $\bar{P}_{\mathrm{n}}$ \\
\hline 4.56 & 0.5 & 0.5 & 0.38 & 300 \\
4.56 & 1.0 & 0.5 & 2.01 & 250 \\
4.56 & 2.0 & 0.5 & 4.74 & 212 \\
4.56 & 4.0 & 0.5 & 7.80 & 144 \\
4.56 & 2.0 & 0.25 & 1.41 & 246 \\
4.56 & 2.0 & 0.13 & 2.25 & 236 \\
4.56 & 2.0 & 0.07 & 3.71 & 225 \\
4.56 & 2.0 & 0.03 & 4.07 & 210 \\
1.52 & 2.0 & 0.5 & 0.42 & 63 \\
2.28 & 2.0 & 0.5 & 1.11 & 85 \\
3.04 & 2.0 & 0.5 & 1.90 & 100 \\
3.80 & 2.0 & 0.5 & 3.28 & 166 \\
\hline
\end{tabular}

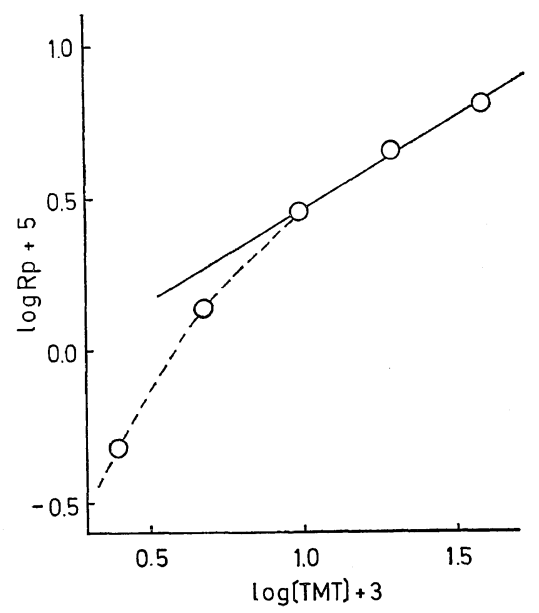

Figure 5. Rate of polymerization $\left(R_{\mathrm{p}}\right)$ vs. concentration of the initiator system in the polymerization of AN with the TMT-DNCB system in $\mathrm{DMF}$ at $80^{\circ} \mathrm{C}$ : [AN], $4.56 \mathrm{~mol} / l,[\mathrm{DNCB}], 0.5 \times$ $10^{-2} \mathrm{~mol} / l ;$ [TMT] was varied. 


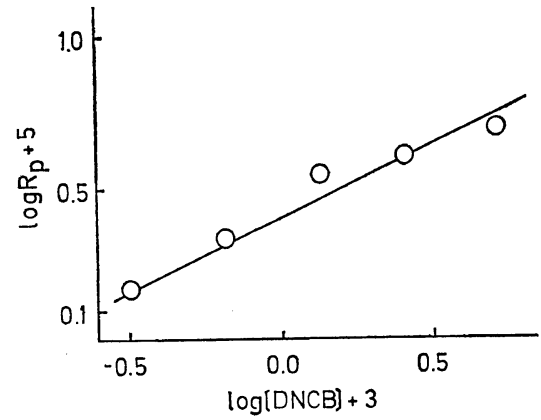

Figure 6. Rate of polymerization $\left(R_{\mathrm{p}}\right)$ vs. concentration of the initiator system in the polymerization of $\mathrm{AN}$ with the TMT-DNCB system in $\mathrm{DMF}$ at $80^{\circ} \mathrm{C}:[\mathrm{AN}], 4.56 \mathrm{~mol} / l ;[\mathrm{TMT}], 2.0 \times$ $10^{-2} \mathrm{~mol} / l$; [DNCB] was varied.

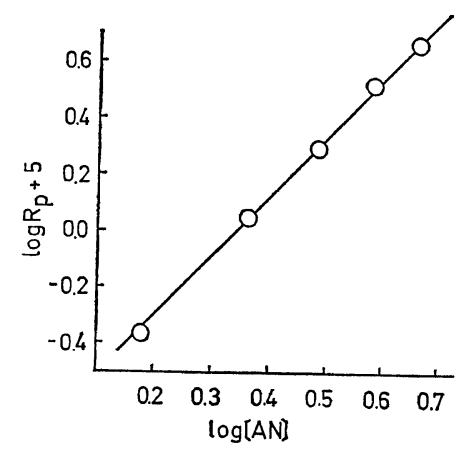

Figure 7. Rate of polymerization $\left(R_{\mathrm{p}}\right) v s$. ANconcentration in the polymerization of $\mathrm{AN}$ with the TMT-DNCB system in DMF at $80^{\circ} \mathrm{C}$ : [TMT], $2.0 \times 10^{-2} \mathrm{~mol} / l ; \quad$ [DNCB], $0.5 \times 10^{-2} \mathrm{~mol} / l ;$ [AN] was varied.

Data in Table I are plotted in Figures 5, 6, and 7. From the figures, the initial rate of polymerization was found to be expressed by the following equation.

$$
R_{\mathrm{p}}=k[\mathrm{TMT}]^{0.60}[\mathrm{DNCB}]^{0.48}[\mathrm{AN}]^{2.00}
$$

\section{The Retardation Effect by DNCB}

In order to check the retardation effect by DNCB in the polymerization of AN with the TMT-DNCB system, we attempted to polymerize $\mathrm{AN}$ in DMF with $\alpha, \alpha^{\prime}$-azobisisobutylonitrile(AIBN) in the presence or absence of DNCB. The results are shown in Figure 8.

The rate of polymerization of $\mathrm{AN}$ by $\mathrm{AIBN}$, was scarcely decreased by the addition of DNCB.

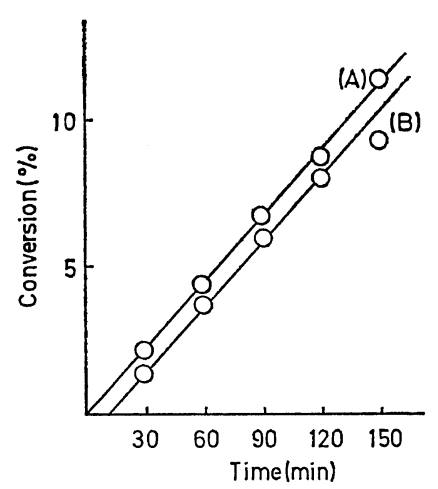

Figure 8. Conversion vs. time, in the polymerization of AN with AIBN in DMF at $52^{\circ} \mathrm{C}$ : line $\mathrm{A},[\mathrm{AN}], 4.56 \mathrm{~mol} / l$, and [AIBN], $0.5 \times 10^{-2} \mathrm{~mol} / l$; line $\mathrm{B},[\mathrm{AN}], 4.56 \mathrm{~mol} / l$, [AIBN], $0.5 \times 10^{-2} \mathrm{~mol} / l$, and [DNCB], $2.0 \times 10^{-2} \mathrm{~mol} / l$.

\section{Estimation of an Overall Activation Energy}

The homogeneous polymerization of AN initiated by the TMT-DNCB system was carried out in DMF, changing the temperature in the range from 60 to $80^{\circ} \mathrm{C}$, where [TMT] and [DNCB] were kept constant at $2.0 \times 10^{-2}$ and $0.5 \times 10^{-2}$ $\mathrm{mol} / \mathrm{l}$, respectively. By an Arrhenius plot of $R_{\mathrm{p}}$ vs. $1 / T$ (Figure 9), the overall activation energy was estimated as $17.8 \mathrm{kcal} / \mathrm{mol}$.

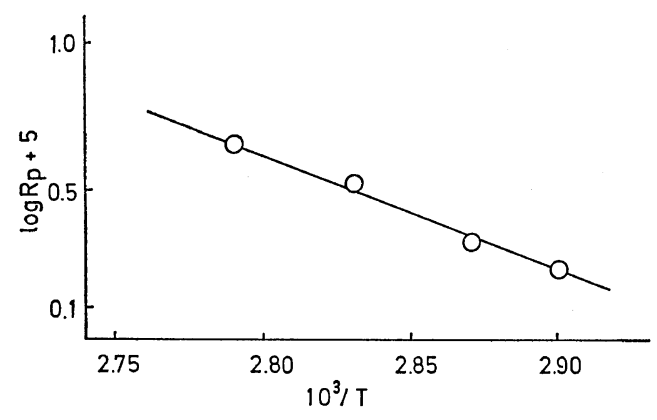

Figure 9. Arrhenius plot of $\log R_{\mathrm{p}}$ vs. $1 / T$.

\section{Molecular Weights of the Polymers Obtained}

The degree of polymerization of the resulting polymers is listed in the Table I and shown in Figure 10.

As can be seen from this figure, the reciprocal of the number-average degree of polymerization approximately depends on the square root of the concentration of TMT and DNCB. 


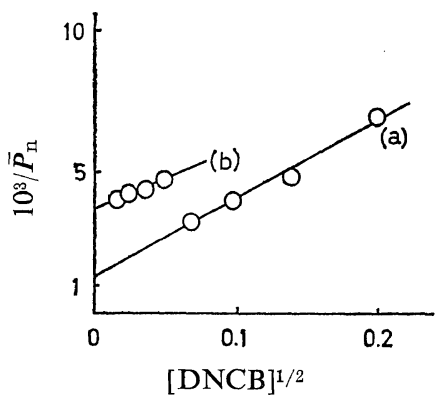

Figure 10. Relationship between $1 / P_{\mathrm{n}}$ and $[\mathrm{TMT}]^{1 / 2}$ or $[\mathrm{DNCB}]^{1 / 2}$ : line (a) $[\mathrm{AN}], 4.56 \mathrm{~mol} / l,[\mathrm{TMT}]$, $2.0 \times 10^{-2} \mathrm{~mol} / l$, and [DNCB] was varied; line (b), [AN], $4.56 \mathrm{~mol} / l, \quad[\mathrm{DNCB}], 0.5 \times 10^{-2} \mathrm{~mol} / l$, and [TMT] was varied.

\section{Estimation of an Equilibrium Constant and Molar Extinction Coefficient of the CT-Band}

In order to clarify the initiation mechanism of the polymerization of AN by the TMT-DNCB system, a closer examination of the interaction between TMT and DNCB in dimethylsulfoxide (DMSO) in the absence of monomer was made by measuring the UV spectra.

TMT has an absorption maximum at $280 \mathrm{~nm}$ in DMSO. There is no absorption of DNCB in the wave-length region from 300 to $500 \mathrm{~nm}$. On the other hand, the situation is very different for a mixed solution of TMT and DMCB in the solvent. As can be seen from Figure 11, two new absorption maxima, due neither to TMT nor DNCB, appear at 376 and $430 \mathrm{~nm}$. The new band at $430 \mathrm{~nm}$ shows a maximum intensity when the molar fraction of TMT is 0.5 in the mixed solution of TMT and DNCB in dimethylsulfoxide(DMSO), as shown in Figure 12. The absorbances in the figure were evaluated by using the average value of the absorbances at 424,430 , and $436 \mathrm{~nm}$.

Therefore, we understand these new bands as being characteristic of the $\sigma$-complex, which was formed as $1: 1$ of TMT and DNCB.

A quantitative consideration of the bands was checked by Benesi-Hildebrand's method. ${ }^{8}$ By the usual procedure, ${ }^{8}$ we evaluated $1.28 \times 10^{4}$ $(376 \mathrm{~nm})$ and $9.21 \times 10^{3}(430 \mathrm{~nm})$ for the molar extinction coefficients of the two bands and 3.34 $\times 10^{2}$ for the equilibrium constant $(K)$ for the complex formation.

On the other hand, the spectral pattern of the

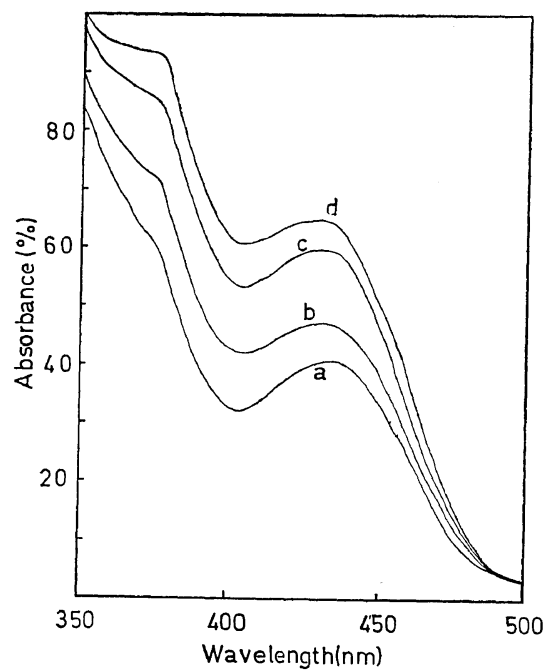

Figure 11. The ultraviolet spectrum of the mixed solution in DMBO. The concentration of DNCB is $9.66 \times 10^{-4} \mathrm{~mol} / l$. The concentration of TMT are $1.49 \times 10^{-4}, 2.99 \times 10^{-4}, 5.97 \times 10^{-4}, 8.96 \times 10^{-4}$, and $1.19 \times 10^{-3} \mathrm{~mol} / l$ for the curves (a), (b), (c), and (d), respectively.

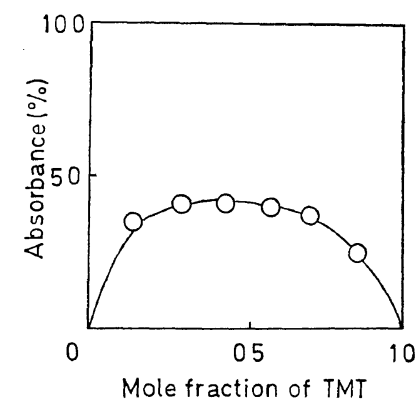

Figure 12. Continuous variation method applied to TMT-DNCB complex.

complex in benzene was substantially similar to that in DMSO, but some loss of the spectral structure occurs, as shown in Figure 13.

Attempted Detections of ESR Signals in the TMT -DNCB System

No ESR signal was obtained in the reaction between TMT and DNCB in DMF, DMSO, or benzene at a degassed state at room temperature.

\section{Reaction of TMT and DNCB}

Six grams $(0.052 \mathrm{~mol})$ of TMT were added to $10 \mathrm{~g}(0.049 \mathrm{~mol})$ of DNCB dissolved in $30 \mathrm{ml}$ of 


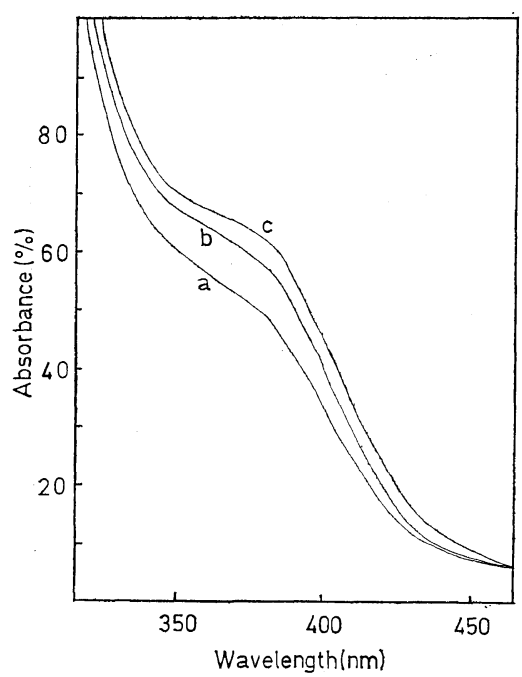

Figure 13. The ultraviolet spectrum of the mixed solution in benzene. The concentration of DNCB is $1.02 \times 10^{-4} \mathrm{~mol} / l$. The concentrations of TMT are $1.97 \times 10^{-3}$, and $3.97 \times 10^{-3} \mathrm{~mol} / l$ for the curves (a), (b), and (c), respectively.

ethanol. After gentle refux for $3 \mathrm{hr}$, the solvent was removed by distillation, and the precipitate was recrystallized several times from ethanol to give $4.5 \mathrm{~g}$ of yellow-crystalline product of $\mathrm{mp}$ $78^{\circ} \mathrm{C}$.

Anal. Calcd for $\mathrm{C}_{8} \mathrm{H}_{9} \mathrm{~N}_{3} \mathrm{O}_{4}: \mathrm{C}, 45.50 ; \mathrm{H}, 4.27$; $\mathrm{N}, 19.91$. Found: C, 45.07; H, 4.06; N, 19.98 . The NMR spectrum (in $\mathrm{CDCl}_{3}$ ) showed at 8.7 ppm $(1 \mathrm{H}), 8.2 \mathrm{ppm}(1 \mathrm{H}), 7.1 \mathrm{ppm}(1 \mathrm{H})$, and 3.1 ppm $(6 \mathrm{H})$. The ultraviolet absorption spectrum (in ethanol) showed $\lambda_{\max } 370 \mathrm{~nm}$.

Thus, this crystalline product was identified as 1-N, $N$-dimethyl-amino-2,4-dinitrobenzene.

\section{DISCUSSION}

As stated above, the order of the effect of the 1-halogeno-2,4-dinitrobenzene on the rate of the polymerization of AN initiated with TMT in DMF was as follows.

$$
\begin{aligned}
\text { F- } \mathrm{C}_{6} \mathrm{H}_{3}\left(\mathrm{NO}_{2}\right)_{2}>\mathrm{Cl}-\mathrm{C}_{6} \mathrm{H}_{3}\left(\mathrm{NO}_{2}\right)_{2} \\
>\mathrm{Br}-\mathrm{C}_{6} \mathrm{H}_{3}\left(\mathrm{NO}_{2}\right)_{2}>\mathrm{I}-\mathrm{C}_{6} \mathrm{H}_{3}\left(\mathrm{NO}_{2}\right)_{2}
\end{aligned}
$$

This order may be better interpreted by the electrophilicity of the carbon atom on the 1-position of the 1-halogeno-2,4-dinitrobenzene rather than by steric hindrance due to the size of the halogen atom.

The polymerization initiated by the system of TMT-1-chloro-2,4-dinitrobenzene(DNCB) was concluded to proceed through a radical mechanism.

The rate of homogeneous polymerization of $\mathrm{AN}$ in DMF was proportional to $[\mathrm{TMT}]^{0.60}$, to $[\mathrm{DNCB}]^{0.48}$, and to $[\mathrm{AN}]^{2.00}$. DMF is known to be a fairly good chain-transfer agent in the radical polymerization of $\mathrm{AN}^{9}$. The value of $C_{S}$ estimated by us was $1.28 \times 10^{-310}$. Considering this fact, the following reaction scheme is proposed.

$$
\mathrm{TMT}+\mathrm{DNCB} \stackrel{K}{\longleftarrow} \text { Complex }(\mathrm{C})
$$

Initiation $\quad \mathrm{C}+\mathrm{M} \stackrel{k_{1}}{\longrightarrow} \mathrm{R}$.

Propagation

$$
\mathbf{R} \cdot+\mathbf{M} \stackrel{k_{2}}{\longrightarrow} \mathbf{R} \text {. }
$$

Chain transfer $\mathrm{R} \cdot+\mathrm{S} \stackrel{k_{3}}{\longrightarrow} \mathrm{RH}+\mathrm{S}$.

Re-initiation with solvent radical

$$
\mathrm{S} \cdot+\mathrm{M} \stackrel{k_{4}}{\longrightarrow} \mathbb{R} \text {. }
$$

Termination

$$
\begin{aligned}
& \text { S. }+ \text { R. } \stackrel{{ }^{k_{5}}}{\longrightarrow} \text { SR (Polymer) } \\
& \text { S. }+ \text { S. } \stackrel{{ }^{k_{6}}}{\longrightarrow} \text { Stabilization } \\
& \text { R. + R } \cdot \stackrel{{ }^{k_{7}}}{\longrightarrow} \text { Polymer }
\end{aligned}
$$

where $M, S$, and $\mathbb{R}$ - are monomer, solvent (DMF), and propagating (or initiating) radical, respectively.

Granting that a stationary state exists, the following expressions would hold.

$$
\mathrm{d}[\mathrm{R} \cdot] / \mathrm{d} t=0, \quad \mathrm{~d}[\mathrm{~S} \cdot] / \mathrm{d} t=0
$$

Accordingly,

$$
\begin{gathered}
k_{1}[\mathrm{C}][\mathrm{M}]+k_{4}[\mathrm{~S} \cdot][\mathrm{M}]=k_{3}[\mathrm{R} \cdot][\mathrm{S}] \\
+k_{5}[\mathrm{~S} \cdot]+k_{7}[\mathrm{R} \cdot]^{2} \\
k_{3}[\mathrm{R} \cdot][\mathrm{S}]=k_{4}[\mathrm{~S} \cdot][\mathrm{M}]+k_{5}[\mathrm{~S} \cdot][\mathrm{R} \cdot]+k_{6}[\mathrm{~S} \cdot]^{2}
\end{gathered}
$$

In eq $11,[\mathrm{M}]$ is much larger than $[\mathrm{S} \cdot]$ or $[\mathrm{R} \cdot]$. Hence, $k_{5}[\mathrm{R} \cdot][\mathrm{S} \cdot]$ and $[\mathrm{S} \cdot]^{2}$ may be negligible, comparing $k_{4}[\mathrm{~S} \cdot][\mathrm{M}]$. Thus the following rate equation was obtained. 
Table II. The overall activation energies of the polymerization of AN initiated by TMT and TMT-promoter systems

\begin{tabular}{ccccccc}
\hline Initiator & TMT & ${\text { TMT }-\mathrm{AA}^{\mathrm{a}}}^{\mathrm{T}}$ & $\mathrm{TMT}^{-\mathrm{BC}^{\mathrm{a}}}$ & $\mathrm{TMT}-\mathrm{DM}^{\mathrm{a}}$ & $\mathrm{TMT}^{-\mathrm{BA}^{\mathrm{a}}}$ & $\mathrm{TMT}-\mathrm{DNCB}$ \\
\hline $\begin{array}{c}E, \\
\mathrm{kcal} / \mathrm{mol}\end{array}$ & 25.4 & 12.6 & 11.4 & 11.9 & 9.4 & 17.8
\end{tabular}

a $\mathrm{AA}, \mathrm{BC}, \mathrm{DM}$, and $\mathrm{BA}$ represent acetic anhydride, benzyl chloride, dimethyl maleate, and bromoacetic acid, respectively.

$$
\begin{aligned}
R_{\mathrm{p}} & =k_{2}[\mathrm{R} \cdot][\mathrm{M}] \\
& =k_{2}\left(k_{1} K\right)^{0.5}[\mathrm{TMT}]^{0.5}[\mathrm{DNCB}]^{0.5}[\mathrm{M}]^{1.5}, \\
& {\left[k_{7}+\frac{k_{3} k_{5}[\mathrm{~S}]}{k_{4}[\mathrm{M}]}\right]^{-0.5} }
\end{aligned}
$$

Eq 12 agrees well with the results obtained in this paper.

It was found that there is an induction period in the polymerization of $\mathrm{AN}$ by the system of TMT and 1-halogeno-2,4-dinitrobenzene. Aromatic nitro-compounds did not retard or inhibit the radical polymerization of $\mathrm{AN}$, as stated by Bagdasaryan, et al. ${ }^{11}$ The existence of the induction period may be a consequence of the period which is neccessary to form the $\sigma$-complex between TMT and DNCB. The over-all activation energies, $E$, of the polymerization of the polymerization of AN initiated with TMTelectrophilic agents are listed in Table II. As an electrophilic agent, DNCB gave the highest value of $E$.

The reaction of TMT with DNCB in the absence of the monomer was found to form a $1: 1$ complex. In the reactions known to involve the breaking of a carbon-halogen bond in the ratedetermining step, the carbon-fluorine bond is usually broken very much slower than other carbon-halogen bonds. ${ }^{12}$ In the nucleophilic displacement of aromatic halides, however, the order of mobility is $\mathrm{F}>\mathrm{Cl}>\mathrm{Br}>\mathrm{I}$ with most alkoxide, mercaptide, and amine reagents. ${ }^{12}$ On the other hand, our results show that the catalytic activities for the polymerization of AN were F- $\mathrm{C}_{6} \mathrm{H}_{3}\left(\mathrm{NO}_{2}\right)_{2}>\mathrm{Cl}-\mathrm{C}_{6} \mathrm{H}_{3}\left(\mathrm{NO}_{2}\right)_{2}>\mathrm{Br}-\mathrm{C}_{6} \mathrm{H}_{3}\left(\mathrm{NO}_{2}\right)_{2}>$ I- $\mathrm{C}_{6} \mathrm{H}_{5}\left(\mathrm{NO}_{2}\right)_{2}$. As mentioned above, our results suggest the participation of monomer in the initiation reaction. That is, the rate is determined by the rate of decomposition of the $\sigma$-complex. The reaction mode and kinetic equation may be as follows

$$
\begin{aligned}
& \mathrm{TMT}+\mathrm{DNCB} \underset{k_{-1}}{\stackrel{k_{1}}{\rightleftarrows}}[\sigma \text {-Complex }] \\
& \stackrel{k_{2}}{\rightleftarrows} \text { Product }+\mathrm{R} .
\end{aligned}
$$

\section{AN}

Applying the steady-state treatment

$$
\begin{aligned}
& \frac{\mathrm{d}[\text { Product }]}{\mathrm{d} t}=\frac{\mathrm{d}[\mathrm{R} \cdot]}{\mathrm{d} t}=\text { Rate } \\
& =\frac{\left(k_{1} k_{2}+k_{1} k_{3}[\mathrm{AN}]\right)[\mathrm{TMT}][\mathrm{DNCB}]}{k_{-1}+k_{2}+k_{3}[\mathrm{AN}]}
\end{aligned}
$$

Since the product-forming (or radical-forming) processes are rate-determining, we can assume $k_{-1} \gg k_{2}+k_{3}[\mathrm{AN}]$, then

$$
\text { Rate }=\frac{k_{1}}{k_{-1}}\left(k_{2}+k_{3}[\mathrm{AN}]\right)[\mathrm{TMT}][\mathrm{DNCB}]
$$

If we assume that $k_{2}<k_{3}[\mathrm{AN}]$ in the polymerization system so that the participation of monomer is expected, we have the following equation.

$$
\text { Rate }=\frac{k_{1}}{k_{-1}} k_{3}[\mathrm{TMT}][\mathrm{DNCB}][\mathrm{AN}]
$$

TMT interacts with DNCB to give a coloured solution. On the nature and structure of the coloured species, we considered several ways by which the interaction took place: ${ }^{13}$ (i) from TMT to DNCB, charge transfer partly occurs; (ii) transfer of an electron from TMT to DNCB to give a radical-anion; (iii) the lone-pair electrons of TMT coordinate with the electrophilic carbon-atom at the 1-position of $\mathrm{DNCB}$, and form a Meisenheimer's adduct. In the present paper, no signals of ESR were observed. Accordingly, the formation of a radical-anion may be excluded. ${ }^{14,15}$ The molar extinction coefficients of the absorption bands of the reaction mixture of TMT and DNCB were $12800(376 \mathrm{~nm})$ and $9210(430 \mathrm{~nm})$, while that of Meisenheimer's 
adduct ${ }^{13}$ has commonly five orders in the 400 $850 \mathrm{~nm}$. Furthermore, the spectral pattern of the reaction mixture favors the Meisenheimer adduct. Accordingly, a Meisenheimer-like adduct, may be formed in the reaction of TMT with DNCB.

From these considerations, the formation of the $\sigma$-complex as a result of an intermolecular reaction of TMT with DNCB was assumed to be reasonable. Thus, the initiation reaction may proceed as follows* mer J., 2, 50 (1971).

5. J. F. Bunnett and R. M. Conner, Org. Syn., 40, 34 (1960).

6. W. E. Bull, J. A. Seaton, and L. F. Audrieth, J. Amer. Chem. Soc., 80, 2516 (1958).

7. R. C. Houts, Text. Res. J., 20, 786 (1950).

8. H. A. Benesi and J. H. Hildebrand, J. Amer. Chem. Soc., 71, 2703 (1949).

9. W. M. Thomas, E. H. Gleason, and J. J. Pellon, J. Polym. Sci., 17, 275 (1955).

10. K. Sugiyama, T. Nakaya, and M. Imoto, submitted to J. Polym. Sci..

$$
\text { -complex }
$$

\section{REFERENCES}

1. J. C. McGowan and L. Seed, Brit. Patatent 808667 (February, 1959); Chem. Abstr., 54, 20329 (1960).

2. T. Nakaya, Y. Maki, and M. Imoto, Makromol. Chem., 113, 131 (1968).

3. T. Nakaya, Y. Maki, and M. Imoto, Makromol. Chem., 125, 161 (1969).

4. K. Sugiyama, T. Nakaya, and M. Imoto, Poly-

* This reaction mode agrees with that of TMT with phthalic anhydride described by Romans. ${ }^{16}$
11. X. S. Bagdasaryan and Z. A. Simizina, J. Phys. Chem. (Russ.) 34, 2736 (1960).

12. J. F. Bunnett and J. J. Randall, J. Amer. Chem. Soc., 80, 6020 (1958).

13. E. Buncel, A. R. Norris, and K. E. Russell, Quart. Rev., 22, 123 (1968).

14. G. A. Russell and E. G. Janzen, J. Amer. Chem. Soc., 89, 300 (1967).

15. A. Ishitani, K. Kuwata, H. Tsubomura, and S. Nagakura, Bull. Chem. Soc. Japan, 36, 1357 (1963).

16. C. J. Michejda and D. Romans, Tetrahedron Lett., 48, 4213 (1969). 